\title{
A rapid identification method of change-point based on field data
}

\author{
Hualin $\mathrm{Xu}^{1, \mathrm{a}}$, Yirui Chen ${ }^{2, \mathrm{~b}}$ \\ ${ }^{1}$ School of Management and Economics, University of Electronic Science and Technology of China, \\ Chengdu 611731, China \\ ${ }^{2}$ Department of Humanities and Social Sciences, Officers' College of PAPF, 610213, China \\ a email: xhl123_ren@126.com, bemail:cyrjamena@163.com
}

Keywords: repairable system; segmented point process model; change point

\begin{abstract}
The repairable system is influenced not only by the depth and breadth of repair, but also by operating conditions, work intensity, mode of operation and many other factors. The failure strength of the system will change once the maintenance effect is significant. And the segmented point process model is a good choice to model this failure-repair process. Existing methods to determine change points or the model calculation are complicated. Therefore, this paper puts forward a new method of modeling change-points. Also, and the validity of this method will be exemplified through case studies.
\end{abstract}

\section{Introduction}

The repairable system refers to a system or a device that can return to the normal state through repair [1]. The reliability of the repairable system in the running process is called operation reliability [2], which is described as failure rate or failure intensity (i.e., the number of failures within the unit time [3]). The repairable system is influenced not only by the depth and breadth of the repair, but also by operating conditions, work intensity, mode of operation and many other factors. When an effect is significant, the failure strength will change. The point that failure intensity changes obviously, is called change point. In the failure process, the constant point process model may result in inaccurate evaluation and repair decision [4], the segmented point process model is a rather good choice, which is first proposed and applied in the medical field. Anderson [5] analyzes the survival rate of cancer patients by the two-stage proportional hazard model, and later, proportional segmentation intensity model has been widely studied and applied in the medical field.

According to the parameter estimation in segmented point process model, Hinkley [6] gives the maximum likelihood estimation having the model parameter of the change point; Carlstein [7] discusses the non-parametric estimation of the change point. Joseph and Wolfson [8] extend the problem of the original single sequence to the problem of multiple sequences, and suggest using empirical Bayes method to estimate change points. If the failure sequence follows a Poisson process, a Poisson process model with change points will be obtained. Raftery and Akman [9] shows Bayes estimation method with one change point in Poisson process; Loschi and Cruz [10]study Bayes estimation method with many change point in Poisson process; Richardson and Basu [11] investigate Bayes estimation method with one change point in the power law process. Existing methods to determine change points or the model calculation are complicated. Therefore, we put forward a new method of modeling change points and exemplify the validity of it through case studies.

\section{The segmented point process model of the repairable system}

If there is a change point in a failure process, and the failure process completes at the point of change, this change point models every failure process with the independent failure point process model. Assume the cut-off time of the failure to observe in the system is $\mathrm{T} ; t_{m}(m=1,2, \ldots n)$ indicates the times of failure within $\mathrm{T}$, and $t_{1}<t_{2}<\ldots<t_{n}$; the change point in the failure process is 
$\tau_{i}(i=1,2, \ldots j)$. Figure1 shows the failure process of the repairable system with changes points. Now let me explain the segmented point process model [4].The model can be extended to multiple change points:

$$
v(t, \phi)=\left\{\begin{array}{ll}
v_{1}\left(t, \phi_{1}\right) & 0 \leq t \leq \tau_{1} \\
v_{i}\left(t-\tau_{i-1}, \phi_{i-1}\right) & \tau_{i-1} \leq t \leq \tau_{i} \\
v_{j+1}\left(t-\tau_{j}, \phi_{j}\right) & \tau_{j} \leq t \leq T
\end{array} \quad i=2,3, \ldots j .\right.
$$

$\tau_{i}(i=1,2, \ldots j)$ tells the change points among the failure process.

\section{Proposed change point modeling method}

\section{Proposed method}

We propose a method to fragment the failure process to determine the change point. For actual observation $\left(t_{1}, t_{2}, \ldots, t_{n}\right)$, we adopt the subsection linear model:

$$
y=\left\{\begin{array}{ll}
a_{1} t+b_{1} & 0 \leq t \leq \tau_{1} \\
a_{i} t+b_{i} & \tau_{1} \leq t \leq \tau_{i} \\
a_{j} t+b_{j} & \tau_{j} \leq t \leq T
\end{array} \quad i=1,2, \ldots j,\right.
$$

We use the least squares method to obtain the parameters of the model, and $\tau_{i}(i=1,2, \ldots j)$ is

$\tau_{i}=\frac{a_{i}-a_{i-1}}{b_{i-1}-b_{i}}$.

The model also needs to solve two key problems:

(1) Estimate the number of failure points $j$ and $\tau_{i}(i=1,2, \ldots j)$ in the failure process .

(2) Take the change point as a boundary, perform fit on the failure point process model, estimate the parameter.

\section{Estimation of parameter}

To the problem (2), we use the maximum likelihood method to estimate parameter. If there is only one change point, cut the failure process into two, and literature [4] gives a likelihood function with one change point.

$$
\ln L[v(t, \phi)]=\sum_{p=1}^{\tau} \ln v\left(t_{p}, \phi_{1}\right)-\int_{0}^{\tau} v\left(t, \phi_{1}\right) d t+\sum_{p=\tau+1}^{n} \ln v\left(t_{p}-\tau, \phi_{2}\right)-\int_{\tau}^{n} v\left(t-\tau, \phi_{2}\right) d t .
$$

$v_{1}(t, \phi)$ and $v_{2}(t, \phi)$ are the failure strength model corresponding to two failure processes; $\phi_{1}$ and $\phi_{2}$ are model parameters

Extend formula (4) to a situation with many change points. We can derive the likelihood function of the subsection point process model with change points:

$$
\ln L[v(t, \phi)]=\sum_{i=1}^{j} \sum_{p=\tau_{i-1}+1}^{\tau_{i}} \ln v_{i}\left(t_{p}-\tau_{i-1}, \phi_{i}\right)-\sum_{i=1}^{j} \int_{\tau_{i-1}}^{\tau_{i}} v_{i}\left(t, \phi_{i}\right) d t+\sum_{p=\tau_{j}+1}^{n} \ln v_{j+1}\left(t_{p}-\tau_{j}, \phi_{j+1}\right)-\int_{\tau_{j}}^{n} v_{j+1}\left(t-\tau_{j}, \phi_{j+1}\right) d t .
$$

$\phi_{i}(i=1,2, \ldots j+1)$ is the model parameter corresponding to $j+1$ section with the boundary $\tau_{i}(i=1,2, \ldots j)$.Here, $T \equiv t_{n}$ is established for the failure of truncated sample.

If $v_{i}(t, \phi)$ is the power law model, formula (5) can be:

$\ln L[v(t, \phi)]=\sum_{i=1}^{j} \sum_{p=\tau_{i-1}+1}^{\tau_{i}} \ln \alpha_{i} \beta_{i}\left(t-\tau_{i-1}\right)^{\beta_{i}-1}-\sum_{i=1}^{j} \int_{\tau_{i-1}}^{\tau_{i}} \alpha_{i} \beta_{i}\left(t-\tau_{i-1}\right)^{\beta_{i}-1} d t+\sum_{p=\tau_{j}+1}^{n} \ln \alpha_{j+1} \beta_{j+1}\left(t-\tau_{j}\right)^{\beta_{j+1}-1}-\alpha_{j+1}\left(t-\tau_{j}\right)^{\beta_{j+1}} d t$. 


\section{Case studies}

\section{Case 1}

We can simulate some failure data as shown in Figure 2.

Applying the sequence detection method, we obtain the likelihood change in the simulation process as shown in Figure 3.

Based on the figure above, we see that there is a change point in the simulation process, and it loses efficacy at the 10th $\tau=2.7121$.

Further, under the method proposed in this paper, we get the change point, model parameter and AIC of the simulation data shown in table 1. Based on this chart, we see that this simulation data has a change point, which is coincident with the result tested by the sequence detection method, but compared with it, our way has less calculation. The sequence detection method needs 17 times of calculation, but this method needs only three.

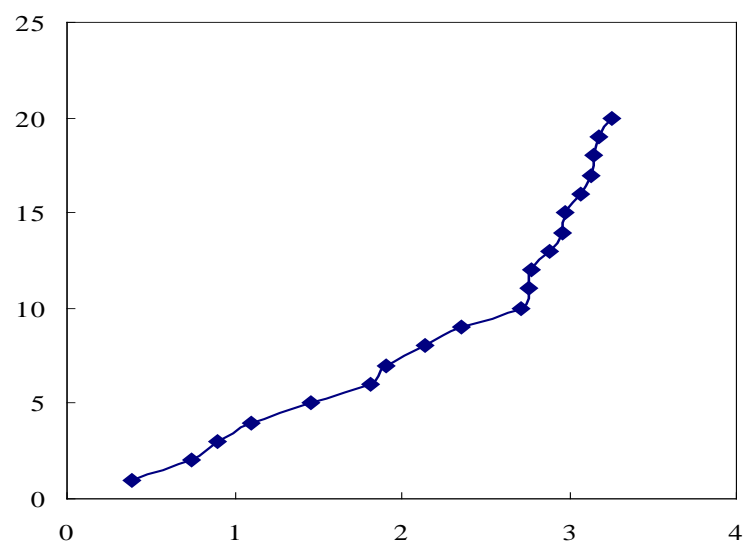

Figure 2 random simulation data

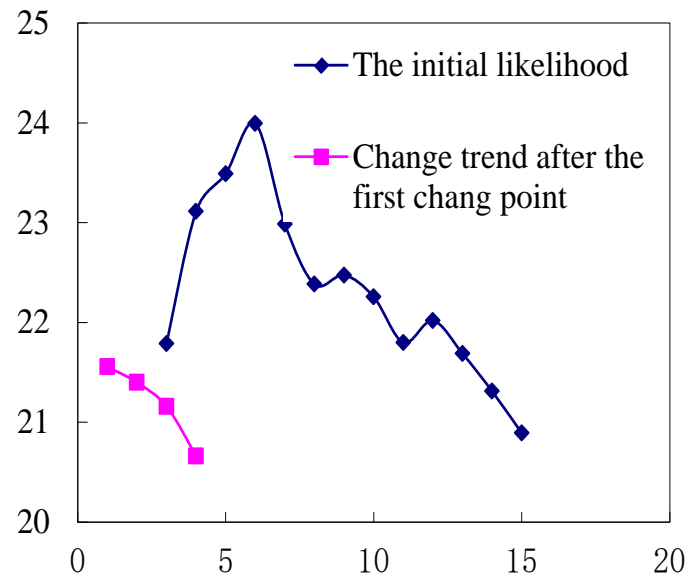

Figure 3 the likelihood change under the sequence detection method

Table 1 the model parameter and AIC

\begin{tabular}{|c|c|c|c|}
\hline & $\tau$ & $\begin{array}{c}\text { The likelihood } \\
\text { value }\end{array}$ & AIC $(j)$ \\
\hline non-change point & - & 25.6404 & 54.9740 \\
\hline One change point & 2.7121 & 22.9855 & 53.9710 \\
\hline Two change points & $0.7435,2.7121$ & 23.5709 & 59.1417 \\
\hline
\end{tabular}

\section{Case 2}

We've collected 216 failure data of vehicle transmission from Sep. 1st, 2006 to Dec. 31st, 2009. These 22 buses run on the same line, have the same operating environment and share similar repair and maintenance. We use the proposed method to determine change points.

According to the method suggested in section 3, we hypothesize here has one and two change points in this failure process, then construct the model by applying the subsection power law point process model. The subsection is shown in table 2 and the estimated parameter and AIC in table 2. 
Table 2 the model parameter and AIC

\begin{tabular}{|c|c|c|c|c|c|}
\hline & \multirow{2}{*}{$\tau$} & \multicolumn{2}{|c|}{ Model parameter } & \multirow{2}{*}{$\begin{array}{l}\text { The likelihood } \\
\text { value }\end{array}$} & \multirow{2}{*}{$\operatorname{AIC}(j)$} \\
\hline & & $\alpha$ & $\beta$ & & \\
\hline $\begin{array}{l}\text { Non-change } \\
\text { point }\end{array}$ & - & 0.0047 & 1.5125 & -573.2549 & 1150.5098 \\
\hline \multirow{2}{*}{$\begin{array}{l}\text { One change } \\
\text { point }\end{array}$} & \multirow{2}{*}{$\tau \approx 952$} & 0.0230 & 1.2584 & \multirow{2}{*}{-563.4045} & \multirow{2}{*}{1136.8090} \\
\hline & & 0.0873 & 1.2365 & & \\
\hline \multirow{3}{*}{$\begin{array}{l}\text { Two change } \\
\text { points }\end{array}$} & \multirow{3}{*}{$\begin{array}{l}\tau_{1} \approx 659 \\
\tau_{2} \approx 995\end{array}$} & 0.0676 & 1.0861 & \multirow{3}{*}{-562.1641} & \multirow{3}{*}{1140.3283} \\
\hline & & 0.2414 & 0.9417 & & \\
\hline & & 0.2369 & 1.0685 & & \\
\hline
\end{tabular}

We can see in table 2 : There is a change point $\tau \approx 952$ in this failure process, which has better goodness-of-fit than non-change point, and we accept the existence of this change point.
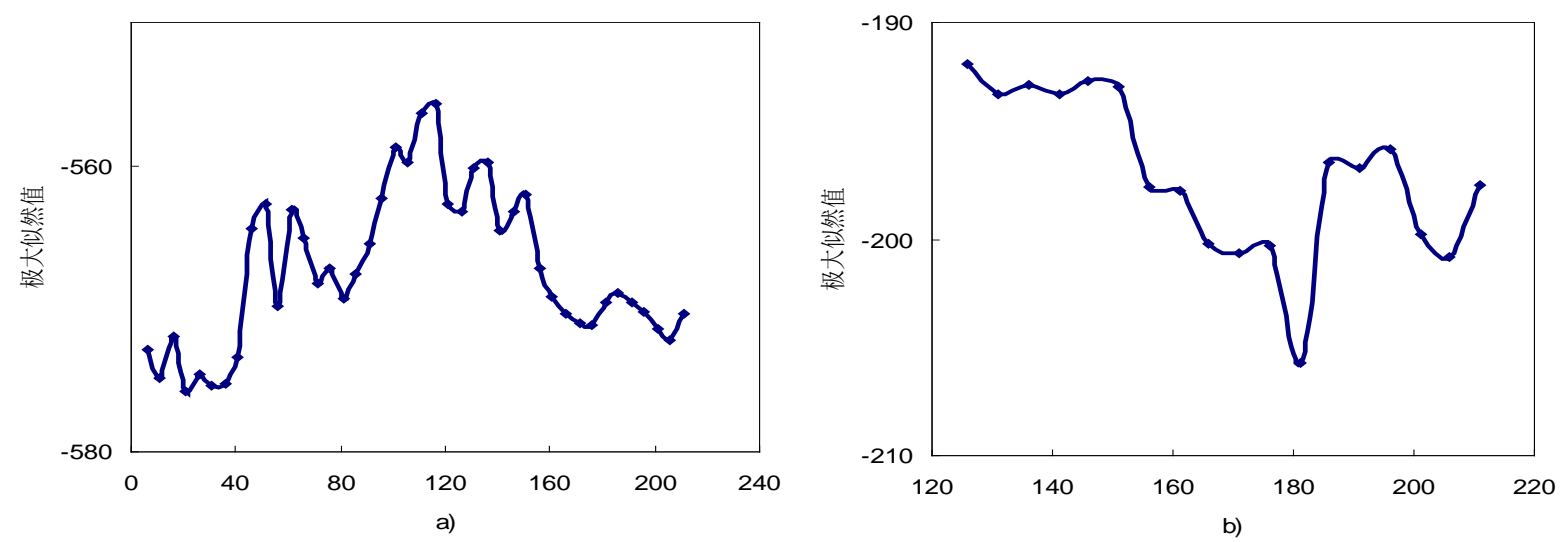

Figure 4 the likelihood value corresponding to the sequence detection method

To make a comparison, we apply the sequence detection method, and observe the change of its likelihood value of the first change point shown in Figure 4 (a) and (b) show the change of its likelihood value of the second change point. In the sequence detection method, we start to calculate from the 6th failure point with the step length 5, and get the change point of the failure process $\tau^{\prime}=929$, very close to the result from the proposed method. After determine the first change point, according to steps of the sequence detection method, we continue to analyze the failure process after the change point, and obtain the trend of the maximal likelihood value in Figure 4 (b), which shows no more change points. This is the same result in this paper.

According to the suggested method, 3 times of calculation are needed to determine the change points in the whole change process, yet, the sequence detection method 42 times when calculate from the 6th failure point with the step length 5, 18 times when start to calculate the 6th failure point after the first change point with the step length 5. Obviously, the method proposed in this paper needs much less calculation.

\section{Summary}

This paper presents a modeling method of change point and the inference process of the method, and illustrates the effectiveness of the proposed method with case study. Compared with the existing methods, this method has the following advantages:

(1) The calculation is far less than the existing methods;

(2) Complete the reliability modeling work when determine the change point.

Additionally, the determination of change point means a lot. After determine the change point in the system failure process, we can construct the failure process in different sections, and can develop repair plans well such as preventive maintenance and repair periodically. 


\section{References}

[1] H. E. Ascher and H. Feingold, Repairable Systems Reliability: Modeling, Inference, Misconceptions and Their Causes. New York: Marcel Dekker, 1984.

[2] D. M. Louit, R. Pascual, and A. K. S. Jardine, "A practical procedure for the selection of time-to-failure models based on the assessment of trends in maintenance data," Reliability Engineering \& System Safety, vol. 94, pp. 1618-1628, 2009.

[3] W. R. Blischke and D. N. P. Murthy, Reliability. New York: Wiley, 2000.

[4] A. Syamsundar and V. N. A. Naikan, "Sequential detection of change points for maintained systems using segmented point process models," Quality and Reliability Engineering International, vol. 25, pp. 739-757, 2009.

[5] J. A. Anderson and A. Senthilselvan, "A Two-Step Regression Model for Hazard Functions," Journal of the Royal Statistical Society. Series C (Applied Statistics), vol. 31, pp. 44-51, 1982.

[6] D. V. Hinkley, "Inference About the Change-Point in a Sequence of Random Variables," Biometrika, vol. 57, pp. 1-17, 1970.

[7] E. Carlstein, "Nonparametric Change-Point Estimation," The Annals of Statistics, vol. 16, pp. 188-197, 1988.

[8] L. Joseph and D. B. Wolfson, "Estimation in multi-path change-point problems." vol. 21: Taylor \& Francis, 1992, pp. 897 - 913.

[9] A. E. Raftery and V. E. Akman, "Bayesian analysis of a Poisson process with a change-point." vol. 73, 1986, pp. 85-89.

[10] R. H. Loschi and F. R. B. Cruz, "Bayesian Identification of Multiple Chang Points in Poisson Data," Advances in Complex Systems, vol. 8, pp. 465-482, 2005.

[11] M. G. Richardson and A. P. Basu, "Inferences on the parameters and system reliability for a failure-truncated power law process: A Bayesian approach using a change-point," 2004, pp. 175-185. 